\title{
The SSP4: A World of Deepening Inequality
}

\begin{abstract}
Five new scenarios, or Shared Socioeconomic Pathways (SSPs), have been developed, spanning a range of challenges to mitigation and challenges to adaptation. The Shared Socioeconomic Pathway 4 (SSP4), "Inequality" or "A Road Divided," is one of these scenarios, characterized by low challenges to mitigation and high challenges to adaptation. We describe, in quantitative terms, the SSP4 as implemented by the Global Change Assessment Model (GCAM), the marker model for this scenario. We use demographic and economic assumptions, in combination with technology and non-climate policy assumptions to develop a quantitative representation of energy, land-use and land-cover, and emissions consistent with the SSP4 narrative. The scenario is one with stark differences within and across regions. High-income regions prosper, continuing to increase their demand for energy and food. Electrification increases in these regions, with the increased generation being met by nuclear and renewables. Low-income regions, however, stagnate due to limited economic growth. Growth in total consumption is dominated by increases in population, not increases in per capita consumption. Due to failures in energy access policies, these regions continue to depend on traditional biofuels, leading to high pollutant emissions. Declining dependence on fossil fuels in all regions means that total radiative forcing absent the inclusion of mitigation or impacts only reaches $6.4 \mathrm{~W} \mathrm{~m}^{-2}$ in 2100 , making this a world with relatively low challenges to mitigation. We explore the effects of mitigation effort on the SSP4 world, finding that the imposition of a carbon price has a varied effect across regions. In particular, the SSP4 mitigation scenarios are characterized by afforestation in the high-income regions and deforestation in the low-income regions. Furthermore, we find that the SSP4 is a world with low challenges to mitigation, but only to a point due to incomplete mitigation of landrelated emissions.
\end{abstract}

\section{Introduction}

The Shared Socioeconomic Pathways (SSPs) were designed to explore a range of challenges to societies in terms of greenhouse gas (GHG) emissions mitigation and adaptation to climate change (O’Neill et al., 2014). The SSPs were designed as reference scenarios, excluding both mitigation and impacts, and thus can facilitate future research into both areas. Within this framework, five different scenarios were articulated (Table 1). SSP1, SSP2, and SSP3 span a range of low, medium, and high challenges to both mitigation and adaptation. SSP5 has high challenges to mitigation, but low challenges to adaptation. Conversely, SSP4 has low challenges to mitigation, but high challenges to adaptation. This last scenario, SSP4, is the focus of this paper. While there are potentially many ways that human societies could find themselves with low challenges to emissions reductions concurrent with major adaptation challenges, the set of circumstances explored in this scenario is outlined in O'Neill, et al. (2015):

Highly unequal investments in human capital, combined with increasing disparities in economic opportunity and political power, lead to increasing inequalities and stratification both across and within countries. Over time, a gap widens between an internationallyconnected society that is well educated and contributes to knowledge-and capital-intensive 
sectors of the global economy, and a fragmented collection of lower-income, poorly educated societies that work in a labor intensive, low-tech economy. Power becomes more concentrated in a relatively small political and business elite, even in democratic societies, while vulnerable groups have little representation in national and global institutions. Economic growth is moderate in industrialized and middle-income countries, while low income countries lag behind, in many cases struggling to provide adequate access to water, sanitation and health care for the poor. Social cohesion degrades and conflict and unrest become increasingly common. Technology development is high in the high-tech economy and sectors. Uncertainty in the fossil fuel markets lead to underinvestment in new resources in many regions of the world. Energy companies hedge against price fluctuations partly through diversifying their energy sources, with investments in both carbon-intensive fuels like coal and unconventional oil, but also low-carbon energy sources. Environmental policies focus on local issues around middle and high income areas. The combination of some development of low carbon supply options and expertise, and a well-integrated international political and business class capable of acting quickly and decisively, implies low challenges to mitigation. Challenges to adaptation are high for the substantial proportions of populations at low levels of development and with limited access to effective institutions for coping with economic or environmental stresses.

SSP4 fits into a class of so-called "barbarization" scenarios in which the negative trends of more conventional, middle of the road scenarios become unmanageable and the gap between the "haves" and "have nots" increases in many parts of the world. The most prominent of these types of scenarios was developed by the Global Scenarios Group (Gallopín, et al., 1997; Electris, et al., 2015). The core theme of barbarization is that extreme poverty, income inequality, and lack of opportunity lead to social and environmental ills, especially for the poor. In one version ("Breakdown"), conflict increases and conditions to lead to "economic collapse". The increased regional rivalry of the SSP3 in some sense reflects this variant. In the "Fortress World" variant of barbarization, an authoritarian response to breakdown emerges, with a class of elites controlling most global resources, similar to that of SSP4. The concentration of power within the elite class, and their ability to trade, in SSP4 results in lower mitigation challenges than in SSP3, because technologies and wealth exist to invest in mitigation, should the will to do so materialize.

The growth explored in SSP4 pattern runs counter to most national and international development goals, for example those promoted through the Millennium Development Goals (United Nations, 2015a) and the new Sustainable Development Goals (United Nations 2015b). That is not to say that some of the characteristics of SSP4 do not appear in the real world today; the theoretical and empirical underpinnings of the SSP4 are discussed in O'Neill, et al. (2015). For example, wealth and income are being increasingly concentrated in many countries, with the gap between rich and poor increasing (OECD, 2015). Inequality results in limited access to education, basic social services, and utilities such as clean water/sanitation for the poor. These and additional characteristics increase the challenges to adaptation in the SSP4 world.

In this paper we describe, in quantitative terms, one way in which SSP4 could unfold over the period to 2100 consistent with the above narrative. We use the Global Change Assessment Model (GCAM) to develop this realization of SSP4 in an internally consistent framework that includes detailed descriptions of the world's energy, economic, and land-use systems. The 
following sections describe in detail our implementation methodology (Section 2), the results without (Section 3) and with climate policy (Section 4), and a comparison of our results to other models and SSPs (Section 5). We conclude with a discussion of the results and future research directions (Section 6). Further information on the implementation of the SSPs in GCAM, as well as additional results are available in the Supporting Online Material. Our analysis focuses on issues related to energy and land use, emissions, climate, and potential mitigation costs. More detailed representation of the aspects of challenges to adaptation is reserved for a future paper.

\section{Methodology}

\subsection{The Global Change Assessment Model (GCAM)}

GCAM couples representations of the economy, the energy system, the agricultural and land use system, and the climate system (Calvin et al., 2011). For the development of the SSPs, we use GCAM4.0 (available at www.globalchange.umd.edu/models/gcam/download), modified to include irrigation (Kim et al., 2016). GCAM, like other IAMs, produces numerical simulations by combining a set of equations that define relationships between model components and parameters, calibrated to historical observations, and driven by a set of external assumptions. Key external assumptions include socioeconomic drivers (e.g., population and GDP), technology characterizations (e.g., cost and efficiency), and policy assumptions, including policies that relate to climate and non-climate policies, regulations and measures.

The GCAM spatial resolution varies hierarchically between model components. GCAM4.0 divides the world into 32 regions for the energy and economy sectors; these regions are further divided for the agricultural and land use sector, resulting in 283 regions. These regions are linked through trade of energy and agricultural commodities, including coal, gas, oil, uranium, bioenergy, and food \& fiber crops. GCAM is a market-equilibrium model; as such, prices are adjusted to ensure supplies and demands of all goods and services are equilibrated in each time step. The model is dynamic-recursive, and thus producers and consumers do not have perfect knowledge of future prices when making decisions. Investment, technology choice, land-use and other decisions that involve long-lived capital and potential future events employ an adaptive expectations assumption; that is, economic decision-makers consider future profit streams but assume that current prices (including $\mathrm{CO}_{2}$ prices) reflect expectations about future prices.

GCAM numerical simulations produce physical and financial outputs, including quantities and prices for various energy and agricultural commodities. For these sectors, GCAM calculates the production, transformation, and final consumption of these commodities. Additionally, the model computes the evolution of the land surface and anthropogenic emissions of 24 GHG and shortlived species.

GCAM is a partial equilibrium model, but it is unique among the IAMs in that its code representations of energy, economy, agriculture, land use, and climate are coupled in a single system, in which all components, including a complete resolution of all component interactions, must be solved simultaneously within a single time period for the model to produce a solution. 


\subsection{Implementing the SSPs in GCAM}

To implement the SSPs within GCAM, we use the demographic and economic assumptions developed by Lutz et al. (2015) and Dellink et al. (2015) in combination with technology and policy assumptions derived from the SSP narratives (O'Neill et al. 2015). Table 1 provides a qualitative summary of these parameters; quantitative details are provided in the Supplementary Material. In general, we have altered parameters related to energy supply (e.g., the capital cost of new power plants, technical change on extraction costs), energy demand (e.g., preferences for traditional bioenergy), agriculture (e.g., agricultural productivity growth), and policy (e.g., start years for carbon policies). We include information on the implementation of the SSP4 (our primary focus), as well as the other SSPs (discussed briefly in Section 5). The SSP4 is characterized by regional differentiation; therefore, we separate our discussion of its implementation and the results of that implementation into income classes (see Table S1 for a mapping of GCAM regions to income classes).

While the SSP4 is also characterized by high degrees of income disparity within regions, we do not explicitly model within-region disparity in this analysis. However, this intraregional inequality does influence the parameterization of the model in some sectors and regions. In particular, we impose higher preferences for fuels and technologies only consumed by the rich or the poor in the SSP4 than in the SSP3 for the same average income. This assumes that a larger portion of the population would fall into the extreme income categories in the presence of intraregional inequality. For the GCAM scenarios, this dynamic is incorporated in the modeling of traditional biomass. Details on the implementation are provided in the Supplementary Material and a further discussion of intraregional inequality is included in Section 6.

As with all SSPs, the influence of climate change on economic decisions is not considered in the SSP4 scenario. This choice was made to facilitate future impacts and adaptation studies that may want to use the SSP4 as a point of departure (see Riahi et al., this issue). A further discussion of the potential effects of climate change on the SSP4 world is included in Section 6.

The SSP4 baseline scenario contains no policies explicitly designed to limit climate change. In Section 4, we discuss the influence of mitigation efforts on an SSP4 world. In particular, we provide replications of the Representative Concentration Pathways (RCPs, van Vuuren et al., 2011), using the SSP4 baseline scenario as a point of departure. The RCPs are defined by their radiative forcing (with units $\mathrm{W} \mathrm{m}^{-2}$ ) in the year 2100. While four RCPs exist (RCP 2.6, RCP 4.5, RCP 6.0, and RCP 8.5), we only include replications of RCP 2.6, RCP 4.5, and RCP 6.0, as the SSP4 baseline does not reach $8.5 \mathrm{~W} \mathrm{~m}^{-2}$. We provide one further mitigation scenario that limits 2100 radiative forcing to $3.4 \mathrm{~W} \mathrm{~m}^{-2}$, as this scenario will be included in CMIP6 (O'Neill et al., 2016).

These replications were created by imposing a set of climate policies, called Shared Climate Policy Assumptions (SPAs; Kriegler, et al., 2014), on the SSP baseline. Each SSP has its own SPA consistent with the narrative from which that SSP was developed. These SPAs describe the policy environment in both the near and long term. In the near term (through 2020), the SPA for SSP4 has regionally differentiated carbon prices, based on Copenhagen pledges, applied to energy and industrial emissions, with no land-related policies. In the long term, the SPA for SSP4 combines a universally applied carbon tax for fossil fuel and industrial emissions limitation 
with a heterogeneous suite of policies and measures for land use change emissions. The SSP4 land use policies are complex (Table 1) and flow from differences in the ability of governments to control land use. It is assumed that in high-income regions (HIRs) governments can successfully implement a policy of afforestation, while in low-income regions (LIRs) governments have less control over land use, hence afforestation policies are assumed to be infeasible. Policy in the middle-income regions (MIRs) falls in between that of HIRs and LIRs; land use is regulated but not perfectly. A detailed, quantitative description of the implementation is provided in Supplemental Material.

\section{The Marker SSP4 Baseline Scenario}

SSP4 is a world of inequality both across and within regions. The underlying socioeconomic scenario assumes large growth in population in LIRs and declining population in MIRs and HIRs. HIRs become increasingly prosperous, while LIRs make little headway in increasing income, leading to an ever-wider per capita income gap (Figure S1). Differences in economic growth across regions lead to vast differences in regional demands for energy and food. In this section, we describe the development pathway of the energy and agricultural systems, as well as changes in land use, emissions, and climate in the SSP4 baseline scenario.

\subsection{Energy}

Energy consumption is driven by the demand for services (heating, cooling, etc.). Service demands for freight and passenger kilometers traveled and residential and commercial floor space (Figure S2) are shown, as indicators of the growth in demand for transportation and building energy services, respectively. These sectors account for 55\% of final energy in 2010 (30\% buildings, 25\% transportation) and 56\% in 2100 (24\% buildings, 32\% transportation); thus, changes in their service demand are good indicators of the growth in energy final demand. Both LIRs and HIRs exhibit limited growth in floor space, but for different reasons. In the HIRs, growth saturates as these regions reach satiation levels. In contrast, growth in per capita demand in the LIRs is limited due to the low-income levels in these regions. While per capita floor space rises in all regions, albeit slowly, global average floor space is stagnant as the fraction of the world's population in LIRs, with lower per capita levels, rises. Growth in transportation services, particularly freight, continues in all regions, such that transportation service in the HIRs is more than four-fold higher than the LIRs in 2100.

Total global final energy grows at a declining rate over the century (Figure 1), effectively stabilizing by 2100. Regional trends, however, are distinctly different. In HIRs and MIRs, final energy use peaks near mid-century and is in decline at the end of the century, as declines in population post-2050 reduce final energy demands despite continued increases in per capita final energy (187 GJ/person/yr in HIRs and $120 \mathrm{GJ} /$ person/yr in medium income countries in 2100). LIRs show a very different pattern, with continued growth throughout the century. This growth is due predominantly to increases in population, as final energy per capita remains low throughout the century (less than $50 \mathrm{GJ} /$ person/yr in 2100).

HIRs rely heavily on electricity to meet their non-transportation energy needs, with electricity supplying $70 \%$ of building energy demand in 2100 . This represents a continued growth from history. For example, electricity accounted for $43 \%$ of United States residential energy use in 2009 (EIA, 2015) and represents 70\% of energy use in 2100 in the SSP4. While there is some 
growth in heating energy demands in HIRs due to increases in floor space and a shift toward electric heating, most of the growth in electricity is for other uses, which includes electronics. Similarly, trends in energy use in LIRs also persist. In these regions, however, traditional biofuels used for heating and cooking remain important in the future, with $35 \%$ of building energy demands met by these fuels in 2100 .

Increases in the demand for electricity are satisfied by a diverse set of fuels (Figure 2) following the SSP4 narrative where energy companies invest in both fossil fuels and low carbon sources. Those investments lead to lower costs (see Tables 1, S2, and S3) and increased electricity generation from low carbon energy sources as the century progresses. For example, nuclear power increases from $12 \%$ in 2010 to $27 \%$ in 2100 and non-biomass renewables increase from $17 \%$ to $42 \%$. By 2100 , these sources supply more than two-thirds of total generation, with only $15 \%$ of electricity generated from coal power and $12 \%$ from natural gas.

Global primary energy consumption nearly doubles in the first half of the century, before declining slightly (Figure 3). Non-fossil energy use increases rapidly through the century, used predominantly for the generation of electricity. Nonetheless, fossil fuels continue to supply more than half of the primary energy mix in 2100 due at least in part to reliance on oil in the transportation sector. The use of oil continues to expand in LIRs throughout the century as population size increases. In HIRs, however, technological progress and declining population post-2050 result in declining consumption. Coal use peaks and declines everywhere, and bioenergy use expands, particularly in LIRs where traditional biomass use continues due to limited economic growth and limited access to modern energy sources.

\subsection{Agriculture and Land Use}

Like the demand for energy services, growth in per capita demand for agricultural goods differs across regions. HIRs again reach satiation levels for crop and livestock demand, limiting the total increase in per capita caloric demand in those regions (Figure S3). Growth in per capita demand in LIRs is also limited, as these regions have limited increases in per capita GDP. The resulting scenario is one with comparable per capita demand for crops between HIRs and LIRs, but HIRs have roughly twice the livestock per capita as LIRs and MIRs. However, as a result of large increases in population in LIRs, total demand for agricultural products eventually surpasses total demand in HIRs.

These demand patterns, coupled with productivity assumptions (Tables 1, S6), are played out in changing global and regional land-use patterns (Figure 4). LIRs increasingly allocate land to pasture and to the production of non-energy crops in order to meet growing crop and livestock demands. Slow growth in productivity of both crop and pasture lands exacerbates the demanddriven land expansion. In contrast, land-use patterns are relatively stable in HIRs where technology improvement remains strong and population pressures are weaker. Water and fertilizer use largely follow trends in crop production, with modest increases in both HIRs and LIRs. Increases in HIRs are positive, but small, as these regions already have high utilization rates; increases in LIRs are positive, but small, as mechanization is incomplete in these regions due to limited economic development. 


\subsection{Emissions}

Global greenhouse gas emissions grow in SSP4 until just after the middle of the century when they peak and begin to decline (Figures 5, S5). These emissions are dominated by fossil fuel and industrial $\mathrm{CO}_{2}$ emissions throughout the century; however, the declining share of fossil fuel use in the energy system dampens future emissions growth. Non-fossil fuel emissions play a larger role in LIRs than in HIRs. LIRs exhibit substantial growth in methane emissions, land-use change $\mathrm{CO}_{2}$, and nitrous oxide $\left(\mathrm{N}_{2} \mathrm{O}\right)$. These emissions in turn can be traced back to factors such as land-use change to expand cultivated area, fertilizer application to expand food and fiber production (producing $\mathrm{N}_{2} \mathrm{O}$ ), and expanded livestock herd size (producing $\mathrm{CH}_{4}$ ) to meet increased food demands predominantly driven by increases in population.

On a global level, the GCAM SSP4 shows limited declines in air pollutant emissions (e.g., sulfur dioxide, nitrogen oxides, black carbon, etc.) over the next century, with emissions in 2100 at approximately $\sim 50 \%$ of their 2010 levels. Pollutant emissions in SSP4, however, exhibit different patterns in low and higher income regions (Figure 6), as the SSP4 narrative suggests a focus of environmental policies on issues in the MIRs and HIRs. While pollutant emissions in HIRs and MIRs decline consistently throughout the century, pollutant emissions in LIRs initially rise and peak near mid-century before declining. The initial rise in emissions is due to growth in the energy sector with limited expansion of pollution controls. As incomes rise, however, LIRs adopt more stringent (though still imperfect) pollution controls leading to the decline in emissions observed in the second half of the century. Some of these emissions (e.g., sulfur dioxide and organic carbon) provide local cooling through aerosol direct and indirect effects, thus their reductions in the latter half of the century provide improved local air quality while unmasking GHG driven climate change.

\subsection{Climate}

The changes in GHG emissions described above drive changes in atmospheric $\mathrm{CO}_{2}$ concentrations, forcing, and temperature in the SSP4 baseline scenario. SSP4 $\mathrm{CO}_{2}$ concentrations rise to slightly more than $700 \mathrm{ppm}$ in the year 2100 (Figure 7). Radiative forcing in SSP4 reaches $6.4 \mathrm{~W} \mathrm{~m}^{-2}$ in 2100 , or approximately $900 \mathrm{ppm} \mathrm{CO}$-equivalent, indicating that non- $\mathrm{CO}_{2}$ emissions contribute the equivalent of an additional $\sim 200 \mathrm{ppm} \mathrm{CO}_{2}$ to climate forcing. Global mean surface temperature change rises almost linearly throughout the century, reaching $\sim 3.7^{\circ} \mathrm{C}$ higher than in the pre-industrial era in 2100.

\section{Mitigation in an SSP4 World}

By definition, the SSP4 baseline scenario excludes both explicit emission mitigation policies and the effect of climate change. In this section, we explore the implications of adding emissions mitigation policies. By comparing scenarios with and without climate policies, the effects of the climate policy can be isolated from the other factors that are changing simultaneously (e.g., population, income, income distribution, land-use). We contrast the SSP4 baseline scenario with four mitigation scenarios, including replications of the RCP 2.6, RCP 4.5, and RCP 6.0 and a fourth scenario that reaches $3.4 \mathrm{~W} \mathrm{~m}^{-2}$ in 2100 . These mitigation scenarios use the SPAs described in Section 2.2. As noted in the Section 2, we cannot replicate the RCP 8.5 with the SSP4 baseline, since its 2100 radiative forcing is less than $8.5 \mathrm{~W} \mathrm{~m}^{-2}$ without climate policy. 
The imposition of climate policy forces reductions in GHG emissions (Figure 5) in an effort to ensure that the radiative forcing targets are met. These policies lead to strong reductions in $\mathrm{CO}_{2}$ emissions (Figure S6), as the non- $\mathrm{CO}_{2} \mathrm{GHG}$ (e.g., $\mathrm{N}_{2} \mathrm{O}$ and $\mathrm{CH}_{4}$ ) mitigation potential in SSP4 is more limited, particularly in LIRs. For brevity we compare the energy and land-use systems in the SSP4 baseline with the SSP4 replication of RCP 2.6 in the following sections, but the full set of SSP4 mitigation scenarios are available in the Supplemental Material.

\subsection{Energy}

Figure 8 shows the change in primary energy use between the SSP4 baseline scenario and the SSP4 RCP 2.6 replication. The SSP4 2.6 requires aggressive emissions mitigation, which is reflected in rapid and fundamental changes in the energy systems of the world, particularly with respect to energy supply (Figure S7). The most prominent change is the rapid reduction in fossil fuel use without $\mathrm{CO}_{2}$ capture and storage (CCS). Coal and gas are used together with CCS through about 2075, but thereafter even this use of coal and gas is phased out. The decline in consumption of fossil fuels is offset by increases in nuclear and renewables, including large-scale deployment of bioenergy with CCS to provide negative emissions.

\subsection{Agriculture and Land Use}

The regionally-differentiated mitigation policy imposed in SSP4 shifts the allocation of land in the SSP4 2.6 (Figure 9), both globally and regionally. Cropland used to grow energy crops increases significantly compared to the SSP4 baseline. This is most notable in low and medium income regions, where bioenergy productivity growth is limited and afforestation policies are absent or incomplete. Afforestation occurs in both medium-income regions (MIRs) and HIRs as economic incentives to sequester carbon are implemented. These increases come at the expense of non-energy crops, pasture and other ecosystems in HIRs and MIRs, while the expansion of bioenergy land in LIRs offsets forests and other ecosystems.

Pressures to expand forests (in MIRs and HIRs) and bioenergy (in LIRs and MIRs) drive up food prices, with a nearly seven-fold increase in producer prices for agricultural commodities in the SSP4 RCP 2.6 replication between 2010 and 2100. These increases in food prices lead to declines in food consumption, a phenomenon observed earlier by Wise et al. (2009).

\subsection{Mitigation Costs}

Carbon prices (Figure S8) remain low in the RCP 6.0 replication, only reaching $\$ 64 / \mathrm{tCO}_{2}$ in 2100. This is not surprising, as SSP4 climate forcing is only modestly higher than the $6 \mathrm{~W} \mathrm{~m}^{-2}$ of the baseline scenario. Prices and costs are also relatively low in the SSP4 RCP 4.5 replication. However, there is a steep increase in costs when the radiative forcing target is reduced from 4.5 $\mathrm{W} \mathrm{m}{ }^{-2}$ to $3.4 \mathrm{~W} \mathrm{~m}^{-2}$ (5-fold increase in 2100 carbon prices), and again when the target is reduced from $3.4 \mathrm{~W} \mathrm{~m}^{-2}$ to $2.6 \mathrm{~W} \mathrm{~m}^{-2}$ (doubling of 2100 carbon price). The SSP4 2.6 ends with a 2100 carbon price that exceeds $\$ 2000 / \mathrm{tCO}_{2}$ and a global policy cost of $8 \%$ of GDP. These increases in carbon prices also drive significant increases in agricultural commodity prices and modest increases in electricity prices in the energy system.

The SSP4 world is one where it is easy to mitigate, but only up to a point. This ease is reflected in the relatively low carbon prices and mitigation costs observed relative to other SSPs for the $4.5 \mathrm{~W} \mathrm{~m}^{-2}$ and $6.0 \mathrm{~W} \mathrm{~m}^{-2}$. As the radiative forcing target declines, however, the cost of mitigation begins to rise significantly. We find that beyond a certain threshold $\left(\sim 2.6 \mathrm{~W} \mathrm{~m}^{-2}\right)$ further 
reductions are no longer possible (see Figure S9) due to the limited ability to reduce land-related GHG emissions in LIRs (see Figure S6).

\section{Comparison to other Quantifications of SSPs}

While GCAM is the "marker" scenario for SSP4, it is one scenario within a larger family of scenarios. Van Vuuren, et al. (2014) observed that replications of SSPs by teams other than the marker team could add useful information. For example, comparing a single SSP with quantifications produced by several models helps illuminate the differences that can be traced to variation in the way models are structured and in the way the qualitative narratives are implemented. Similarly, a set of SSPs produced by a single model provides a way of isolating the effects of variation in the SSP narrative from variation across models. In this section, we compare the GCAM SSP4 to (1) quantifications of the SSP4 by the AIM (Fujimori, et al., this issue) and WITCH (Riahi, et al., this issue) models and (2) to other SSPs, using both the SSP marker scenarios and the GCAM replications of those scenarios. In the interest of space, we limit our discussion to a comparison of the baseline scenarios, without mitigation efforts. A discussion of mitigation in the other GCAM SSPs is included in the Supplementary Material. Additional discussion of how the SSP4 fits in the broader SSP family is provided in Riahi et al. (this issue), as well as information on energy in Bauer et al. (this issue), land in Popp et al. (this issue), and local air pollution in Rao et al. (this issue).

The GCAM SSP4 baseline has a radiative forcing that is $0.5-0.7 \mathrm{~W} \mathrm{~m}^{-2}$ higher in 2100 than either the AIM or WITCH replication (Figure 10), as AIM and WITCH have lower emissions of GHGs than the GCAM baseline. For example, GCAM estimates cumulative fossil fuel and industrial $\mathrm{CO}_{2}$ emissions of $\sim 4300 \mathrm{GtCO}_{2}$ between 2010 and 2100, compared to $\sim 3300 \mathrm{GtCO}_{2}$ in AIM and $\sim 3900 \mathrm{GtCO}_{2}$ in WITCH. These are due primarily to differences in fossil fuel consumption. All three models estimate that fossil fuel consumption will peak around midcentury, but differ in the peak value (750 $\mathrm{EJ} \mathrm{yr}^{-1}$ in GCAM, $650 \mathrm{EJ} \mathrm{yr}^{-1}$ in WITCH, and $~ 500$ $\mathrm{EJ} \mathrm{yr}^{-1}$ in AIM, Figure S16). This peak is the result of an increasing share of non-fossil fuels, as total primary energy increases monotonically in all models.

The SSP4 has lower radiative forcing in the year 2100 than SSP2, SSP3 and SSP5, regardless of the IAM used (Figure 10). For example, the GCAM SSP5 reaches $8.4 \mathrm{~W} \mathrm{~m}^{-2}$ in 2100, compared to $8.7 \mathrm{~W} \mathrm{~m}^{-2}$ in the REMIND-MAgPIE SSP5 marker and the $6.4 \mathrm{~W} \mathrm{~m}^{-2}$ in the GCAM SSP4 marker. Differences between SSP4 and SSP5 radiative forcing are largely due to differences in $\mathrm{CO}_{2}$ emissions. The SSP5 has significantly larger fossil fuel and industrial $\mathrm{CO}_{2}$ emissions than SSP4 despite lower population, due to higher per capita energy demand and a higher share of fossil fuels. These dynamics are true when comparing the SSP4 to both the GCAM SSP5 and marker SSP5 scenarios.

At the other end of the spectrum, the SSP4 has higher radiative forcing than either the GCAM SSP1 (5.6 $\mathrm{W} \mathrm{m}^{-2}$ in 2100) or the IMAGE SSP1 marker $\left(5.0 \mathrm{~W} \mathrm{~m}^{-2}\right.$ in 2100). These differences are due largely to differences in land-use change $\mathrm{CO}_{2}, \mathrm{CH}_{4}$ and $\mathrm{N}_{2} \mathrm{O}$, where the SSP4 has $\mathrm{CH}_{4}$ and $\mathrm{N}_{2} \mathrm{O}$ emissions nearly double that of either the GCAM SSP1 or the IMAGE SSP1 in 2100 (Figure S18). Differences in land-related emissions are due to assumptions about per capita food demand and intensification of the agricultural sector. The SSP1 has smaller growth in per capita 
livestock demand due to preferences for sustainability and higher growth in agricultural productivity particularly in the LIRs.

The SSP3 and SSP4 are similar in some elements of the narrative, with large low income populations. However, differences in inequality between the two SSPs lead to different results, both in GCAM and the other IAMs. First, inequality between regions in the SSP4 results in a global elite that invests in energy technology. The result is lower cost low-carbon energy technologies and consequently higher deployment (30\% in GCAM SSP4-Baseline, 50\% in AIM SSP4-Baseline, compared to $\sim 15 \%$ in both GCAM and AIM SSP3-Baseline). Second, inequality between regions in the SSP4 leads to higher use of traditional bioenergy for the same average income level than in the SSP3. For example, at a global average income of $\sim \$ 11,500$, GCAM estimates an average of 3.2 GJ per person of traditional bioenergy in the SSP3, compared to 4.4 GJ per person at a comparable income level in the SSP4. Finally, differences in population globally and income in the MIRs and HIRs result in differences in global energy and food consumption, both per capita and total. The SSP4 has higher per capita values, as the MIRs and HIRs bring up the global average; but the SSP3 has higher total values as population far exceeds that of SSP4 (See Figures S1, S16, and S17).

\section{Discussion}

The Shared Socioeconomic Pathway 4 (SSP4), "Inequality" or "A Road Divided," is one of the five SSPs developed to guide the creation of new scenarios for the "Parallel Process" (Moss et al., 2010). This GCAM quantification of the SSP4, described in this paper, is a world with stark differences across regions. These differences play out in terms of demand for energy and food, as well as the means of meeting that demand. HIRs prosper, continuing to increase their per capita demand for energy and food. Electrification increases in these regions, with the increased generation being met by nuclear and renewables. Per capita demand in LIRs, however, stagnates due to limited growth in income. Continued growth in population, however, results in increases in total demand in these regions. Energy poverty in LIRs is high, with per capita final energy demand remaining below 50 GJ per person. These regions continue to depend on traditional biofuels, leading to high pollutant emissions. LIRs also have increases in crop and pastureland to meet food demands for a growing population with limited technological progress and limited trade. Due to a declining dependence on fossil fuels in all regions, total radiative forcing, absent any effort to mitigate, only reaches $6.4 \mathrm{~W} \mathrm{~m}^{-2}$ in 2100 .

We have used this baseline scenario as a foundation from which to create RCP replications for RCPs 2.6, 4.5, and 6.0, as well as a scenario that limits climate forcing to $3.4 \mathrm{~W} \mathrm{~m}^{-2}$ in 2100 . Under efforts to mitigate, the regional differentiation becomes more pronounced, particularly on the land surface. HIRs allocate more land to forests in an effort to store more carbon, offsetting energy-related emissions. LIRs, however, deforest, shifting land toward the production of energy crops. The pressures of land-based mitigation options lead to a substantial increase in food prices. We found that the SSP4 may be a world with relatively low challenges to mitigation for modest climate targets, but the regional heterogeneity of income, technology, and land-based mitigation potential results in steep increases in the cost of meeting stricter climate targets, such as the RCP 2.6. 
As a result of the inequality within the SSP4 scenario, the ability to achieve sustainable development goals is regionally differentiated. HIRs make progress toward eradicating hunger, improving air quality, and providing energy access. LIRs, however, continue to struggle to meet these goals. With the imposition of a climate policy, progress is also made on limiting climate change. This last goal is met with fewer challenges than are present in the other SSPs.

The quantifications of the SSP4 described in this paper are contingent on the realization of the SSP4 narrative used. Important research questions that follow from this effort include: under which circumstances will such a world emerge, and is the continuation and deepening of such inequality plausible? For example, would rich elites be able to maintain their wealth and status, given that extreme inequality could lead to lower overall economic growth (Ostry, et al. 2014) and increase social dissolution, worsening class relations, resulting in large-scale political conflict and civil unrest (Kawachi and Kennedy 1997; Mutaner and Lynch 1999).)? Current trends, such as increases in the economic prominence of financial services, would tend to concentrate incomes in the upper classes and reduce the earnings of workers with less education who provide labor to industry or other less prominent sectors, lending credence to the potential for the scenario to be realized. However, as increasing resources are likely to be required to maintain order and protect the wealth and power of the elites, the SSP4 could evolve into something more akin to the "breakdown" world of SSP3.

The inequality described in the SSP4 world will have major implications for the distribution of climate change impacts and the resources available to the world's impoverished majority to adapt. In this paper, we do not focus on the implications for climate change impacts and adaptation but rather examine the challenges associated with mitigation. However, given the narrative, future analyses of the effects of climate change impacts and adaptation (or lack thereof) in the SSP4 world are necessary and interesting.

Additionally, other potential improvements are possible to the work that we have reported here. One of the most obvious is to develop national and regional models that include income distributions within countries and regions. Our analysis paid special attention to inequality across regions, but only considered inequality within individual nations and regions through parameterizations of preferences for traditional bioenergy. Further consideration is difficult within an integrated assessment model, but is important to consider as these scenarios are downscaled for use at local scales. Furthermore, these dynamics could have significant implications for impacts and adaptation.

In the future, our analysis could be enhanced with an explicit consideration of hydrology and its interactions with energy and agricultural systems in all SSPs. The GCAM scenarios account for water demand for agricultural uses, but do not explicitly include constraints on the expansion of that water use. Additionally, we do not compute water demands for other sectors of the economy in this analysis. Finally, the degree, nature, and geospatial distribution of climate change remain subject to substantial uncertainty. Future work can benefit from more explicit treatment of uncertainty within and across the SSPs. For example, a systematic exploration of the implications of uncertainty in key variables would be insightful. Such a study would help illuminate the factors that drive challenges to mitigation and challenges to adaptation. 


\section{References}

Bauer et al. (this issue). "Shared Socio-Economic Pathways of the Energy Sector - Quantifying the Narratives." Global Environmental Change.

Calvin, K., L. Clarke, J. Edmonds, J. Eom, M. Hejazi, S. Kim, P. Kyle, R. Link, P. Luckow and P. Patel. (2011). "GCAM Wiki Documentation." from https://wiki.umd.edu/gcam/.

Crespo, J., Income projections for climate change research: A framework based on human capital dynamics. Global Environ. Change (2015), http://dx.doi.org/10.1016/j.gloenvcha.2015.02.012

Dellink, R., et al., Long-term economic growth projections in the Shared Socioeconomic Pathways. Global Environ. Change (2015), http://dx.doi.org/10.1016/j.gloenvcha.2015.06.004

EIA (Energy Information Administration) 2015. Table CE2.1 Household Site Fuel Consumption in the U.S., Totals and Averages, 2009, https://www.eia.gov/consumption/residential/data/2009/c\&e/ce2.1.xlsx

Electris, Christi, P. Raskin, R. Rosen, and J. Stutz (2015). The Century Ahead: Four Global Scenarios. Technical Documentation. Tellus Institute: Boston, MA, USA.

Fujimori, Shinichiro, et al. (2015) SSP3: AIM Implementation of Shared Socioeconomic Pathways. This volume.

Gallopin, G., A. Hammond, P. Raskin, and R. Swart. 1997. Branch Points: Global Scenarios and Human Choice: A Resource Paper of the Global Scenario Group. PoleStar Series Report No. 7. Stockholm Environment Institute: Stockholm, Sweden.

Hartin, C. A., Patel, P., Schwarber, A., Link, R. P., and Bond-Lamberty, B. P.: A simple objectoriented and open-source model for scientific and policy analyses of the global climate system Hector v1.0, Geosci. Model Dev., 8, 939-955, doi:10.5194/gmd-8-939-2015, 2015.

Kawachi, I., and P.B. Kennedy. 1997. "Health and social cohesion: why care about income inequality?" BMJ : British Medical Journal 314(7086): 1037-1040.

Kim, Son H., Mohamad Hejazi, Lu Liu, Kate Calvin, Leon Clarke, Jae Edmonds, Page Kyle, Pralit Patel, Marshall Wise, and Evan Davies. 2015. Balancing Global Water Availability and Use at Basin Scale in an Integrated Assessment Model. Accepted for publication in Climatic Change.

Kriegler, E., Edmonds, J., Hallegatte, S., Ebi, K. L., Kram, T., Riahi, K., Winkler, H. \& Van Vuuren, D. P. 2014. A new scenario framework for climate change research: The concept of shared climate policy assumptions. Climatic Change, 122, 401-414.

Jiang, L., O’Neill, B.C., Global urbanization projections for the Shared Socioeconomic Pathways. Global Environ. Change (2015), http://dx.doi.org/10.1016/j.gloenvcha.2015.03.008 
Leimbach, M., et al., Future growth patterns of world regions - A GDP scenario approach. Global Environ. Change (2015), http://dx.doi.org/10.1016/j.gloenvcha.2015.02.005

Lutz, KC, S., W., The human core of the shared socioeconomic pathways: Population scenarios by age, sex and level of education for all countries to 2100. Global Environ. Change (2014), http://dx.doi.org/10.1016/j.gloenvcha.2014.06.004

Moss, R.; J. Edmonds, K. Hibbard, T. Carter, S. Emori, M. Kainuma, T. Kram, M. Manning, J. Meehl, J. Mitchell, N. Nakicenovic, K. Riahi, S Rose, S.J. Smith, R. Stouffer, A. Thomson, D. vanVuuren, J. Weyant, and T. Wilbanks. 2010. "A New Approach to Scenario Development for the IPCC Fifth Assessment Report," Nature, 463:747-756, (11 February 2010) doi:10.1038/nature08823.

Mutaner, C., and J. Lynch. 1999. "Income inequality, social cohesion, and class relations: a critique of Wilkinson's neo-Durkheimian research program.” Int J Health Serv. 1999;29(1):59-81.

O’Neill, B., E. Kriegler, K. Riahi, K. Ebi, S. Hallegatte, T. Carter, R. Mathur and D. van Vuuren (2014). "A new scenario framework for climate change research: the concept of shared socioeconomic pathways." Climatic Change 122(3): 387-400.

O’Neill, Brian C., Elmar Kriegler, Kristie L. Ebi, Eric Kemp-Benedict, Keywan Riahi, Dale S. Rothman, Bas J. van Ruijven et al. "The roads ahead: Narratives for shared socioeconomic pathways describing world futures in the 21st century." Global Environmental Change (2015). doi:10.1016/j.gloenvcha.2015.01.004

Ostry, Jonathan D., A. Berg, and C.G. Tasangarides. 2014. Redistribution, Inequality, and Growth. International Monetary Fund. Washington, DC.

www.imf.org/external/pubs/ft/sdn/2014/data/sdn1402.zip

Popp et al. (this issue). "Shared Socio-economic Pathways - a framework for assessing potential land use futures." Global Environmental Change.

Rao et al. (this issue). "Future Air Pollution in the Shared Socio-Economic Pathways." Global Environmental Change.

Riahi et al. (this issue). “The Shared Socioeconomic Pathways: An Overview.” Global Environmental Change.

Shinichiro Fujimori, Tomoko Hasegawa, Toshihiko Masui, Kiyoshi Takahashi, Diego Silva Herran, Hancheng Dai, Yasuaki Hijioka, and Mikiko Kainuma, Global Environ. Change (2016), SSP3: AIM Implementation of Shared Socioeconomic Pathways, this Special Issue.

Thomson, Allison M., Katherine V. Calvin, Steven J. Smith, G. Page Kyle, April Volke, Pralit Patel, Sabrina Delgado-Arias, Ben Bond-Lamberty, Marshall A. Wise, Leon E. Clarke and James 
A. Edmonds. 2011. "RCP 4.5: A Pathway for Stabilization of Radiative Forcing by 2100," Climatic Change, Special Issue on Representative Concentration Pathways. 109:77-94. DOI 10.1007/s10584-011-0151-4.

United Nations. 2015a. The Millennium Development Goals Report 2015. United Nations. New York. ISBN 978-92-1-101320-7.

United Nations. 2015b. Transforming our world: the 2030 Agenda for Sustainable Development. Adopted by the U.N. General Assembly. United Nations. New York. http://undocs.org/A/RES/70/1.

Van Vuuren D, JA Edmonds, M Kainuma, K Riahi, AM Thomson, KA Hibbard, G Hurtt, T Kram, V Krey, JF Lamarque, Matsui, M Meinhausen, N Nakicenovic, SJ Smith, and SK Rose. 2011. "The Representative Concentration Pathways: An Overview." Climatic Change. 109:531.

Van Vuuren, D. P., Kriegler, E., O'Neill, B. C., Ebi, K. L., Riahi, K., Carter, T. R., Edmonds, J., Hallegatte, S., kram, T., Mathur, R. \& Winkler, H. 2014. A new scenario framework for Climate Change Research: Scenario matrix architecture. Climatic Change, 122, 373-386.

Van Vuuren, Detlef P. , Elke Stehfest, David Gernaat, Jonathan Doelman, Maarten van Berg, Mathijs Harmsen, Harmen-Sytze de Boer, Lex Bouwman, Vassilis Daioglou, Oreane Edelenbosch, Bastien Girod, Tom Kram, Luis Lassaletta, Paul Lucas, Hans van Meijl, Christoph Müller, Bas van Ruijven, Andrzej Tabeau (2016). Energy, land-use and greenhouse gas emissions trajectories under a green growth paradigm. Global Environ. Change, this Special Issue

Wise, M., K. Calvin, A. Thomson, L. Clarke, B. Bond-Lamberty, R. Sands, S.J. Smith, A. Janetos, and J. Edmonds. 2009. Implications of Limiting CO2 Concentrations for Land Use and Energy. Science 324:1183-1186. [PNNL-17943]. 
Tables and Figures

Table 1: Qualitative Assumptions for the GCAM SSPs

\begin{tabular}{|c|c|c|c|c|c|c|c|c|}
\hline & & \multirow[b]{2}{*}{ SSP1 } & \multirow[b]{2}{*}{ SSP2 } & \multirow[b]{2}{*}{ SSP3 } & \multicolumn{3}{|c|}{ SSP4 } & \multirow[b]{2}{*}{ SSP5 } \\
\hline & & & & & $\begin{array}{l}\text { High } \\
\text { Income }\end{array}$ & $\begin{array}{l}\text { Medium } \\
\text { Income }\end{array}$ & Low Income & \\
\hline \multirow[t]{2}{*}{ Socioeconomics } & Population in 2100 & 6.9 billion & 9 billion & 12.7 billion & 0.9 billion & 2.0 billion & 6.4 billion & 7.4 billion \\
\hline & $\begin{array}{l}\text { GDP per capita in } \\
2100\end{array}$ & $\$ 46,306$ & $\$ 33,307$ & $\$ 12,092$ & $\$ 123,244$ & $\$ 30,937$ & $\$ 7,388$ & $\$ 83,496$ \\
\hline \multirow{3}{*}{$\begin{array}{l}\text { Fossil Resources } \\
\text { (Technological } \\
\text { Change/Acceptance) }\end{array}$} & Coal & Med/Low & Med/Med & High/High & Med/Low & Med/Med & Med/High & High/High \\
\hline & $\begin{array}{l}\text { Conventional Gas \& } \\
\text { Oil }\end{array}$ & Med/Med & Med/Med & Med/Med & High/Low & High/Low & High/Low & High/High \\
\hline & Unconventional Oil & Low/Med & Med/Med & Med/Med & Med/Low & Med/Low & Med/Low & High/High \\
\hline \multirow{3}{*}{$\begin{array}{l}\text { Electricity } \\
\text { (Technology Cost) }\end{array}$} & Nuclear & High & Med & High & Low & Low & Low & Med \\
\hline & Renewables & Low & Med & High & Low & Low & Low & Med \\
\hline & $\mathrm{CCS}$ & High & Med & Med & Low & Low & Low & Low \\
\hline \multirow[t]{2}{*}{ Fuel Preference } & Renewables & High & Med & Med & High & High & High & Med \\
\hline & Traditional Biomass & Low & Low & High & Low & Low & High & Low \\
\hline \multirow{3}{*}{$\begin{array}{l}\text { Energy Demand } \\
\text { (Service Demands) }\end{array}$} & Buildings & Low & Med & Low & High & Med & Low & High \\
\hline & Transportation & Low & Med & Low & High & Med & Low & High \\
\hline & Industry & Low & Med & Low & High & Med & Low & High \\
\hline \multirow{5}{*}{$\begin{array}{l}\text { Agriculture \& Land } \\
\text { Use }\end{array}$} & Food Demand & High & Med & Low & High & Med & Low & High \\
\hline & Meat Demand & Low & Med & High & Med & Med & Med & High \\
\hline & Productivity Growth & High & Med & Low & High & Med & Low & High \\
\hline & Trade & Global & Global & Global & Regional & Regional & Local & Global \\
\hline & SPA* Policy & Afforestation & $\begin{array}{l}\text { Limited } \\
\text { afforestation }\end{array}$ & No land policy & $\begin{array}{l}\text { Afforestatio } \\
\mathrm{n}\end{array}$ & $\begin{array}{l}\text { Limited } \\
\text { afforestation }\end{array}$ & $\begin{array}{l}\text { No land } \\
\text { policy }\end{array}$ & Afforestation \\
\hline Pollutant Emissions & Emissions Factors & Low & Med & High & High & High & High & Low \\
\hline \multirow[t]{2}{*}{ Marker } & Model & IMAGE & $\begin{array}{l}\text { MESSAGE- } \\
\text { GLOBIOM }\end{array}$ & AIM & \multicolumn{3}{|c|}{ GCAM } & $\begin{array}{l}\text { REMIND- } \\
\text { MAGPIE }\end{array}$ \\
\hline & Reference & $\begin{array}{l}\text { Van Vuuren } \\
\text { et al. (this } \\
\text { issue) } \\
\end{array}$ & $\begin{array}{l}\text { Riahi et al. } \\
\text { (this issue) }\end{array}$ & $\begin{array}{l}\text { Fujimori et al. } \\
\text { (this issue) }\end{array}$ & \multicolumn{3}{|c|}{ This Paper } & $\begin{array}{l}\text { Kriegler et al. } \\
\text { (this issue) }\end{array}$ \\
\hline \multirow[t]{2}{*}{ Challenges } & $\begin{array}{l}\text { Challenges to } \\
\text { Mitigation }\end{array}$ & Low & Medium & High & \multicolumn{3}{|c|}{ Low } & High \\
\hline & $\begin{array}{l}\text { Challenges to } \\
\text { Adaptation }\end{array}$ & Low & Medium & High & \multicolumn{3}{|c|}{ High } & Low \\
\hline
\end{tabular}




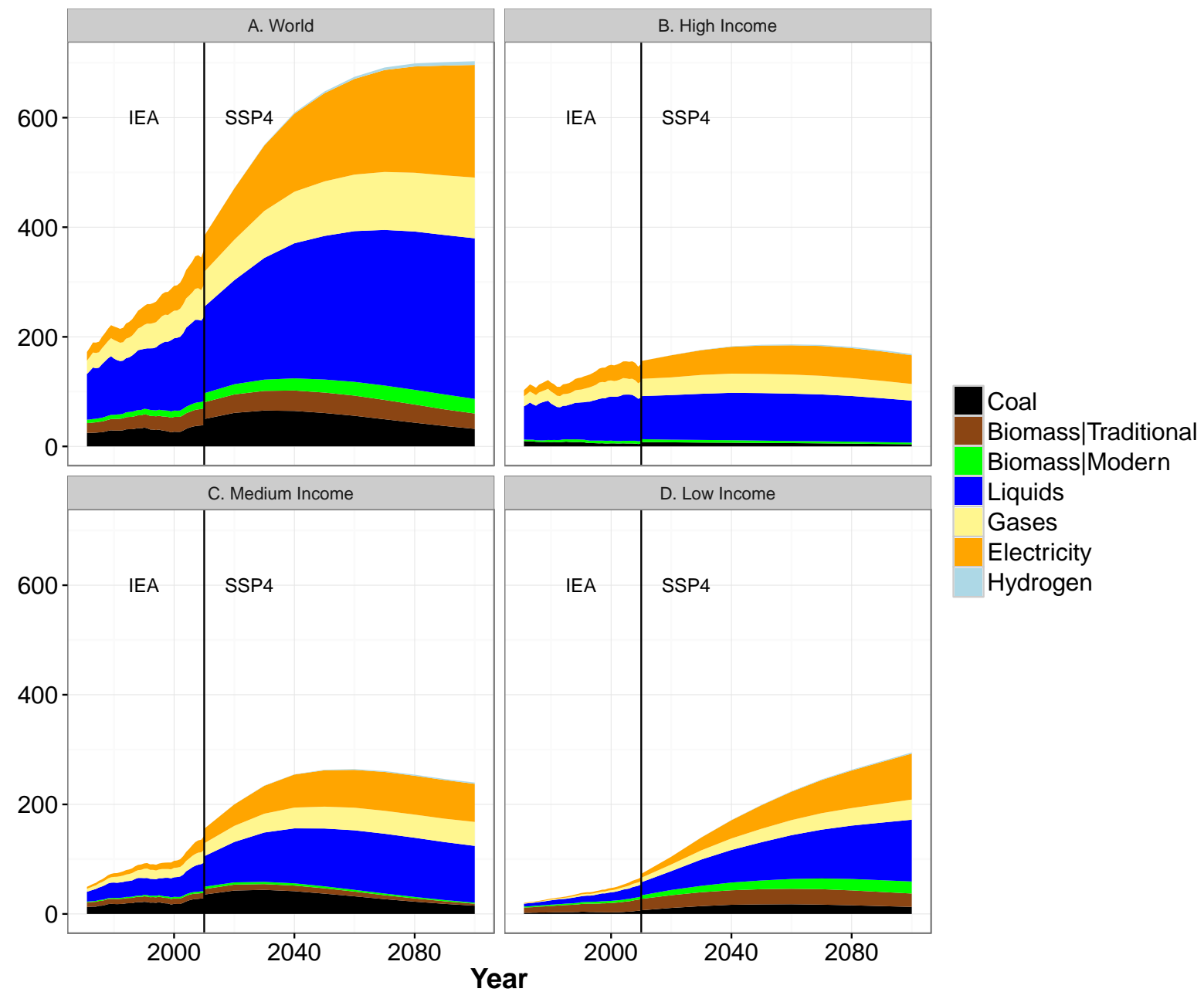

Figure 1: Final Energy Demand by Income Group, both Historically (from IEA) and in the SSP4 Baseline. High Income regions have per capita GDP greater than or equal to $\$ 12,275$ per person (2010\$). Low Income regions have GDP per capita less than $\$ 2,750$ per person (2010\$). A mapping from GCAM region to income group is provided in the Supplementary Material. Final energy differs between GCAM and the IEA in 2010 due to a difference in how energy is aggregated across transformation sectors and end-use sectors. More information is provided in the supplementary material. 

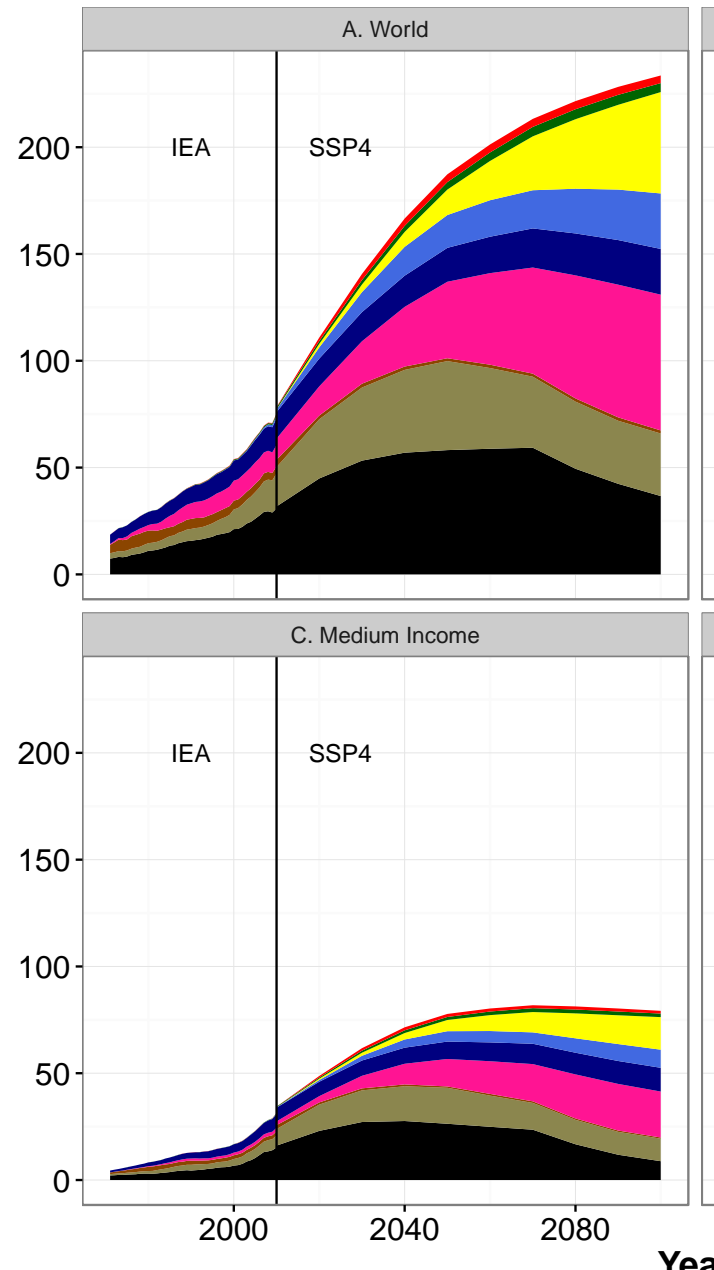

Year
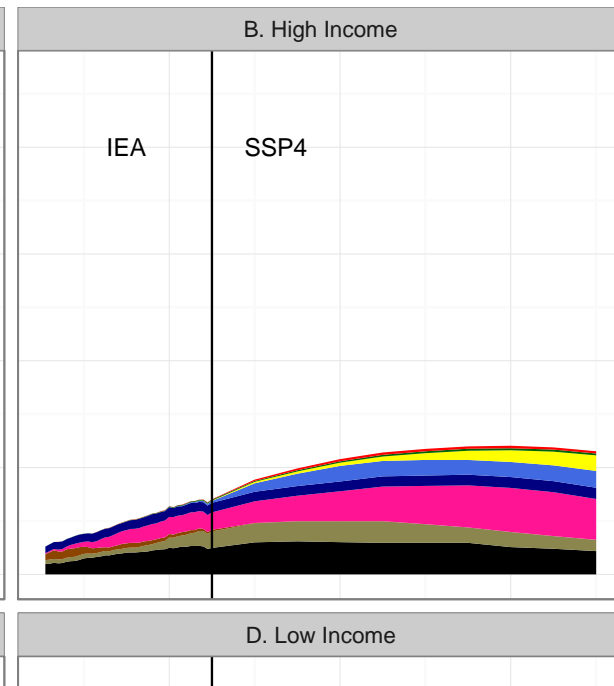

IEA

SSP4

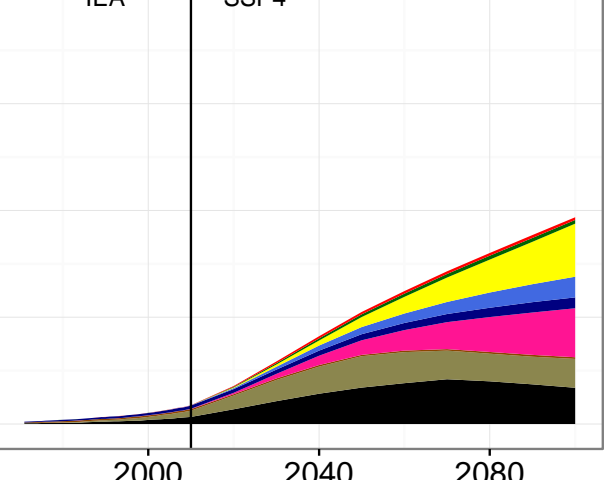

Coal

Gas

Oil

Nuclear

Hydro

Wind

Solar

Biomass

Geothermal

Figure 2: Electricity Generation by Income Group, both Historically (from IEA) and in the SSP4 Baseline.

Electricity generation differs between GCAM and the IEA in 2010 due to a difference in how energy is aggregated across transformation sectors and end-use sectors. More information is provided in the supplementary material. 

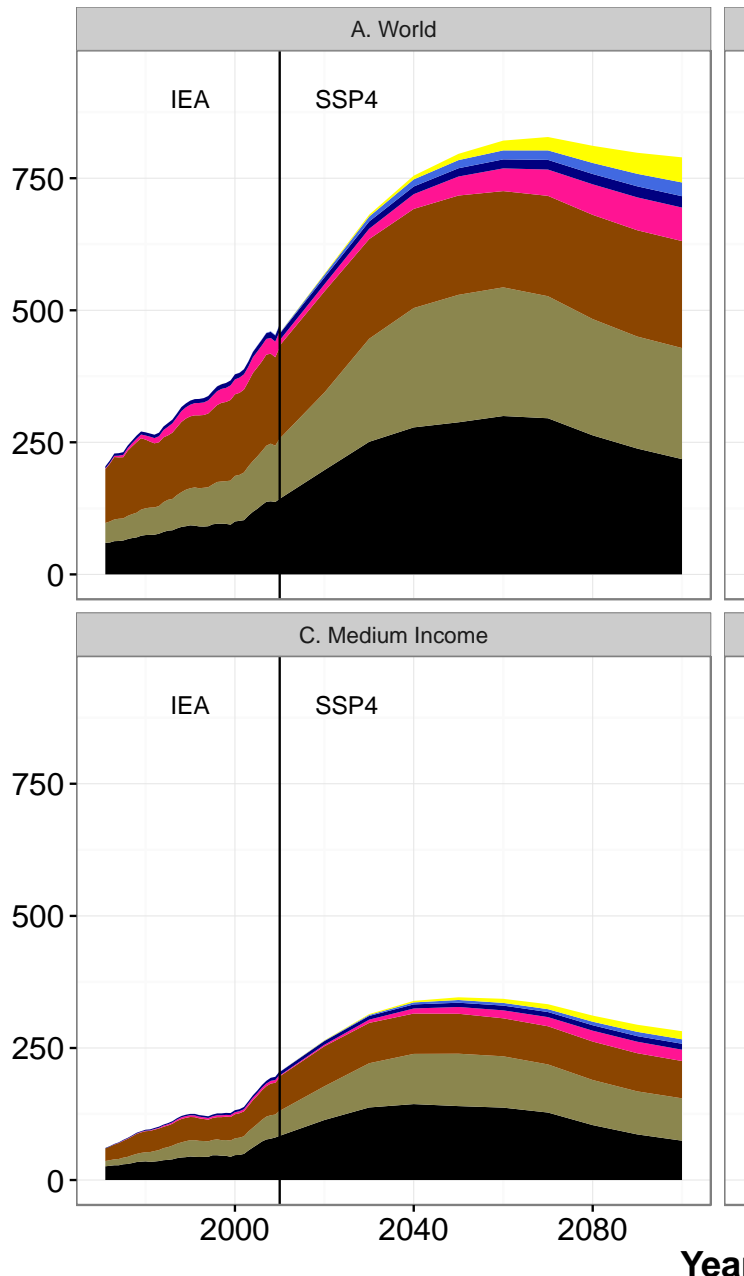

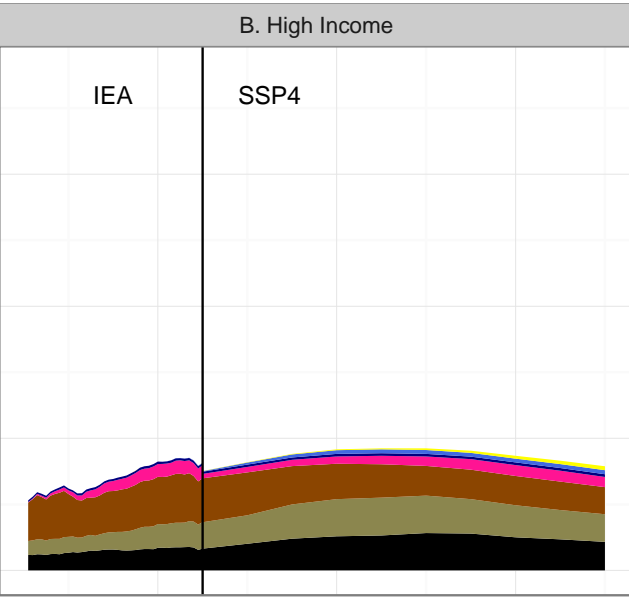

D. Low Income

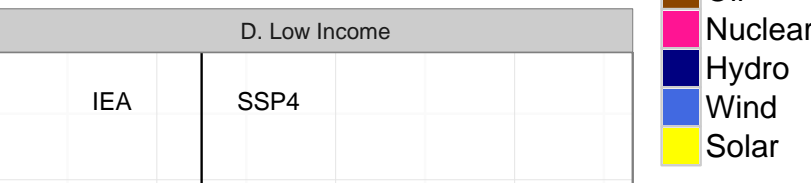

Year
Coal

Gas

Oil

Nuclear

Hydro

Solar

Figure 3: Primary Energy Consumption by Income Group, both Historically (from IEA) and in the SSP4 Baseline 


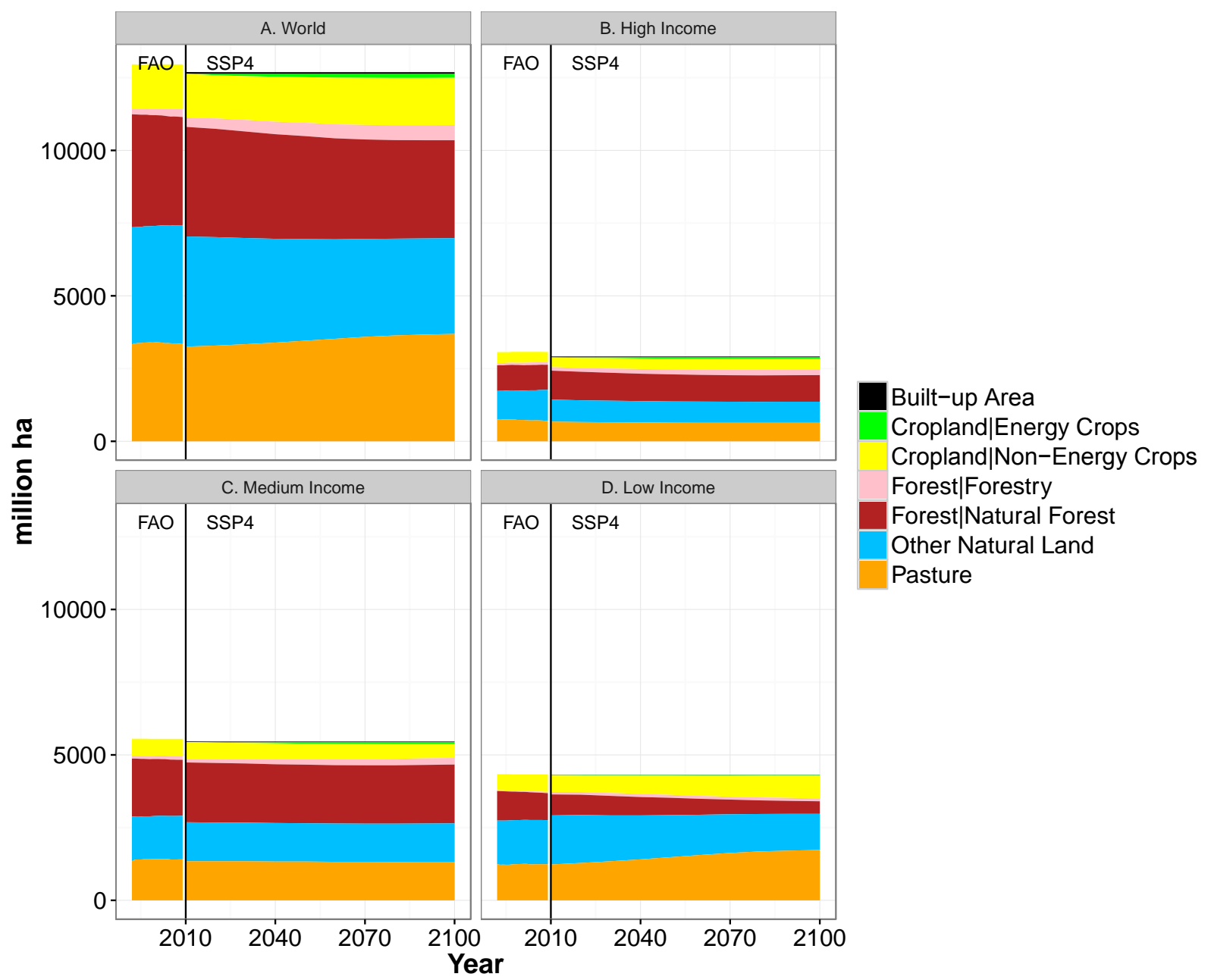

Figure 4: Land Cover by Income Group, both Historically (from FAO) and in the SSP4 Baseline. Land cover data in GCAM is derived from a blend of data sources (see Supplementary Material) and thus does not match FAO land cover data in 2010. 


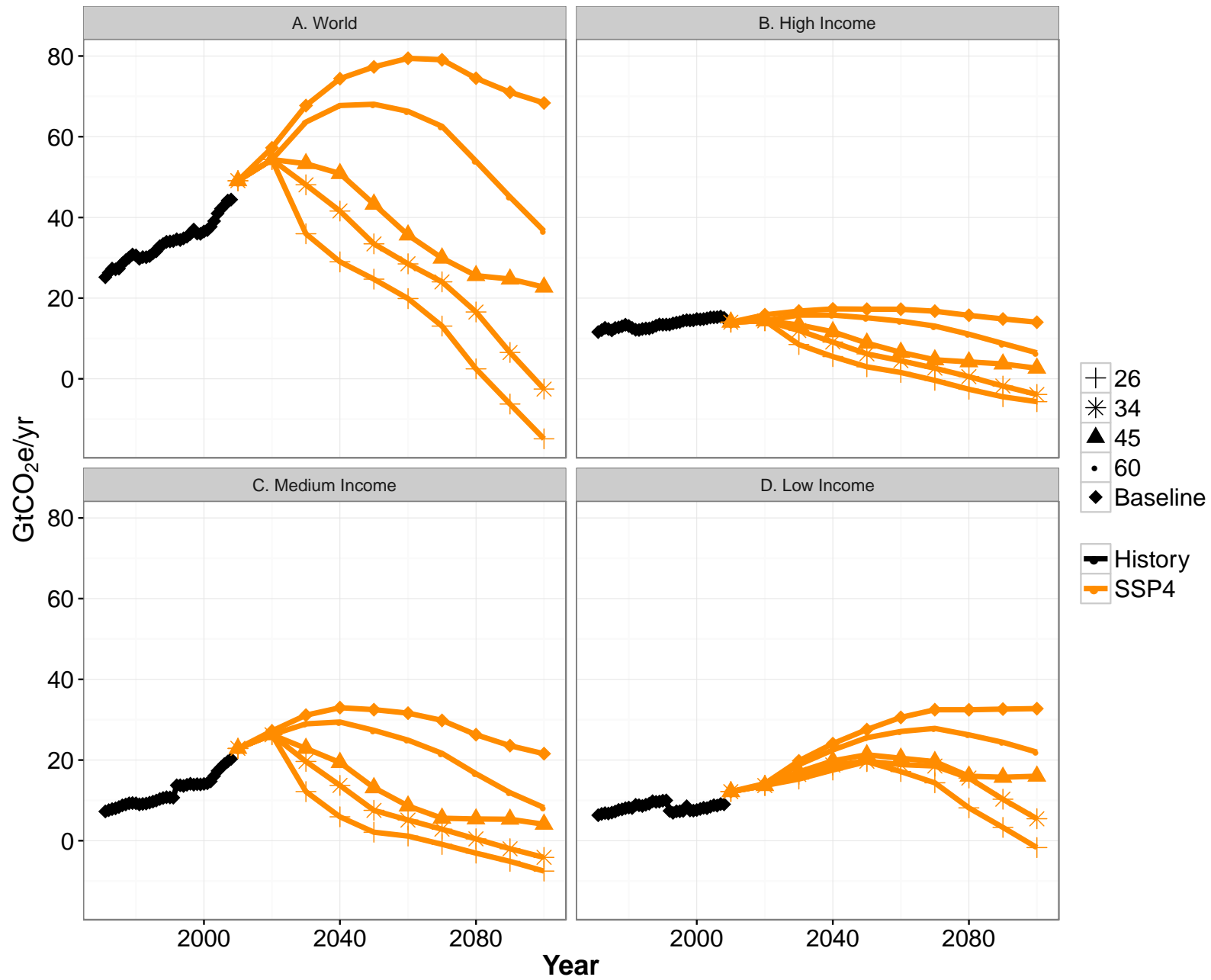

Figure 5: Greenhouse Gas Emissions by Income Group, both Historically ( $\mathrm{CO}_{2}$ from CDIAC and Non- $\mathrm{CO}_{2}$ from EDGAR) and in the SSP4 Baseline and Mitigation Cases 

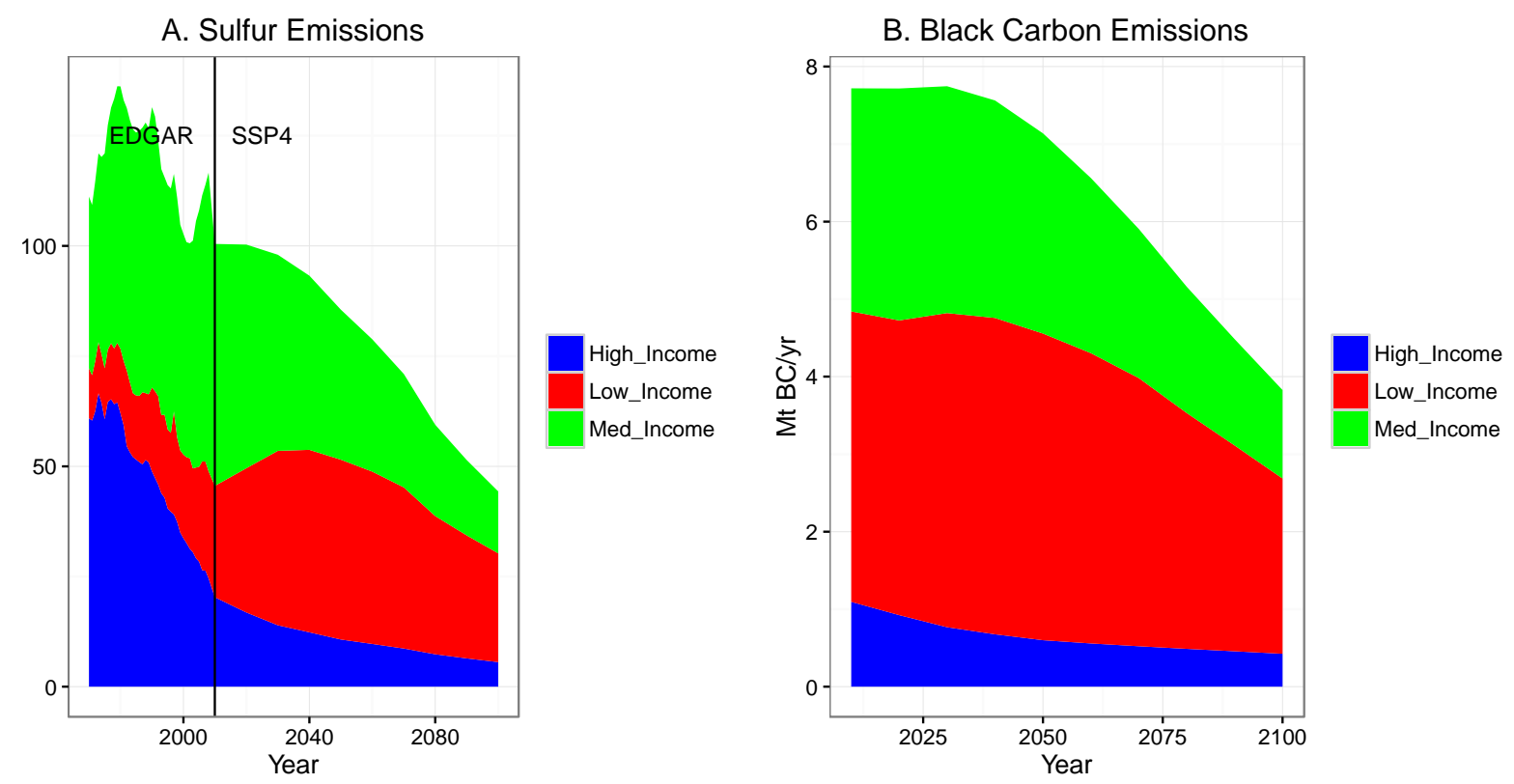

Figure 6: Pollutant Emissions by Income Group, both Historically (from EDGAR) and in the SSP4 Baseline 
A. $\mathrm{CO}_{2}$ Concentration

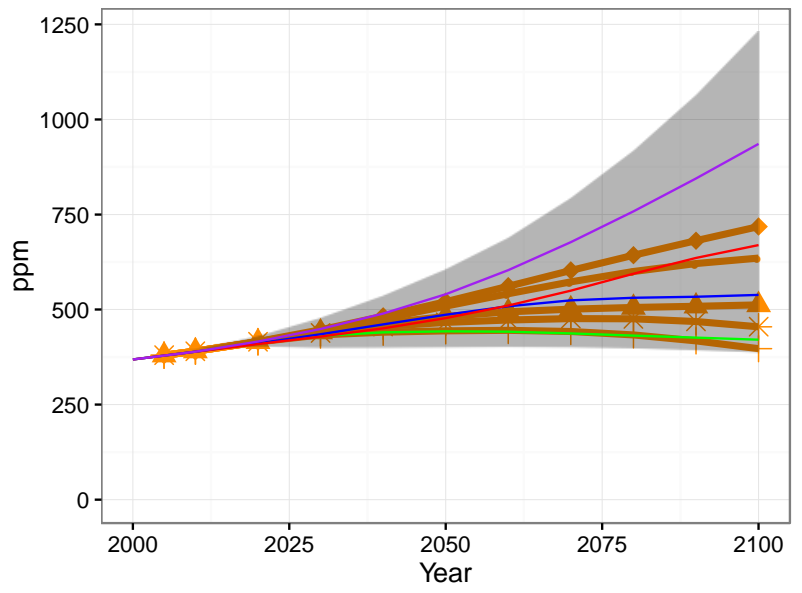

C. Global Mean Temperature Rise

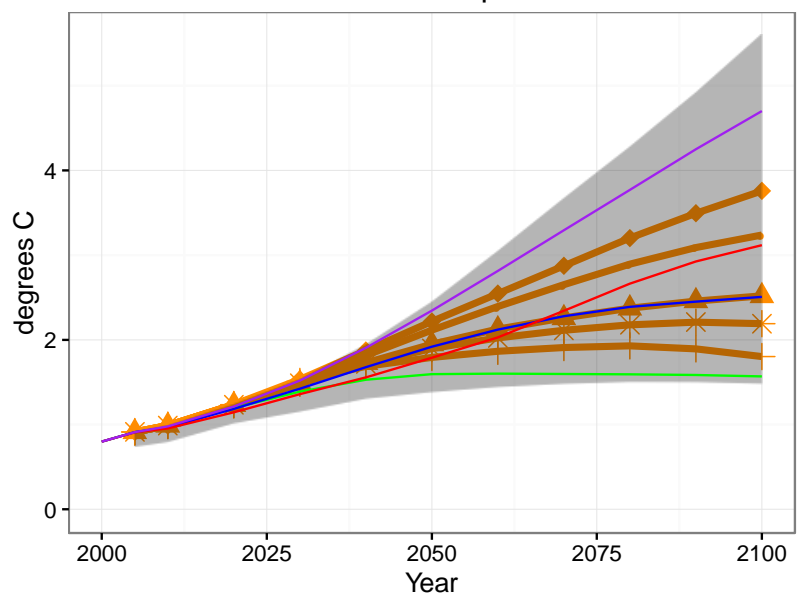

B. Forcing

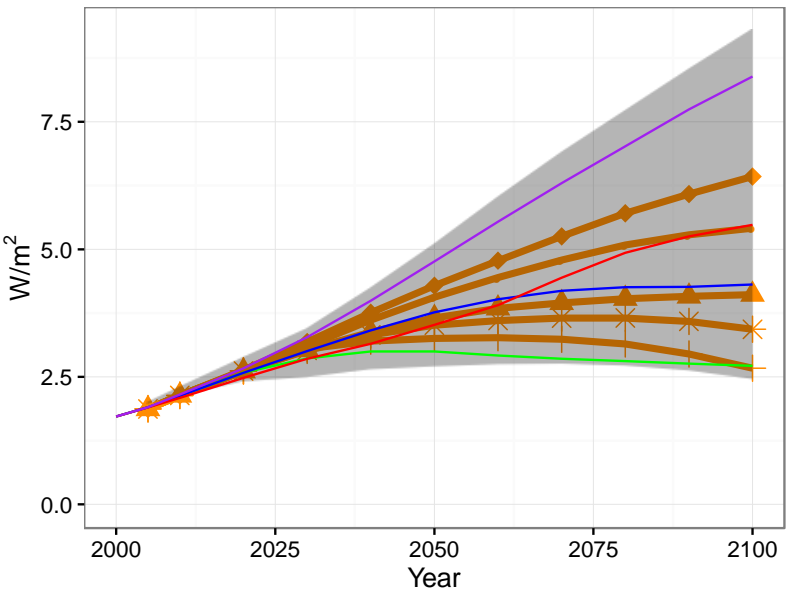

$\mathrm{RF}$

$+26$

* 34

- 45

- 60

- Baseline

SSP

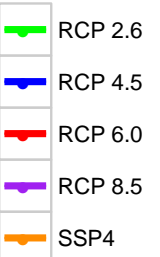

Figure 7: Climate Information for the SSP4 Baseline and Mitigation Scenarios (orange lines), the RCPs (red, green, blue, and purple lines) and the range of the IPCC $5^{\text {th }}$ Assessment Report (gray shaded area). Results are from MAGICC6. 


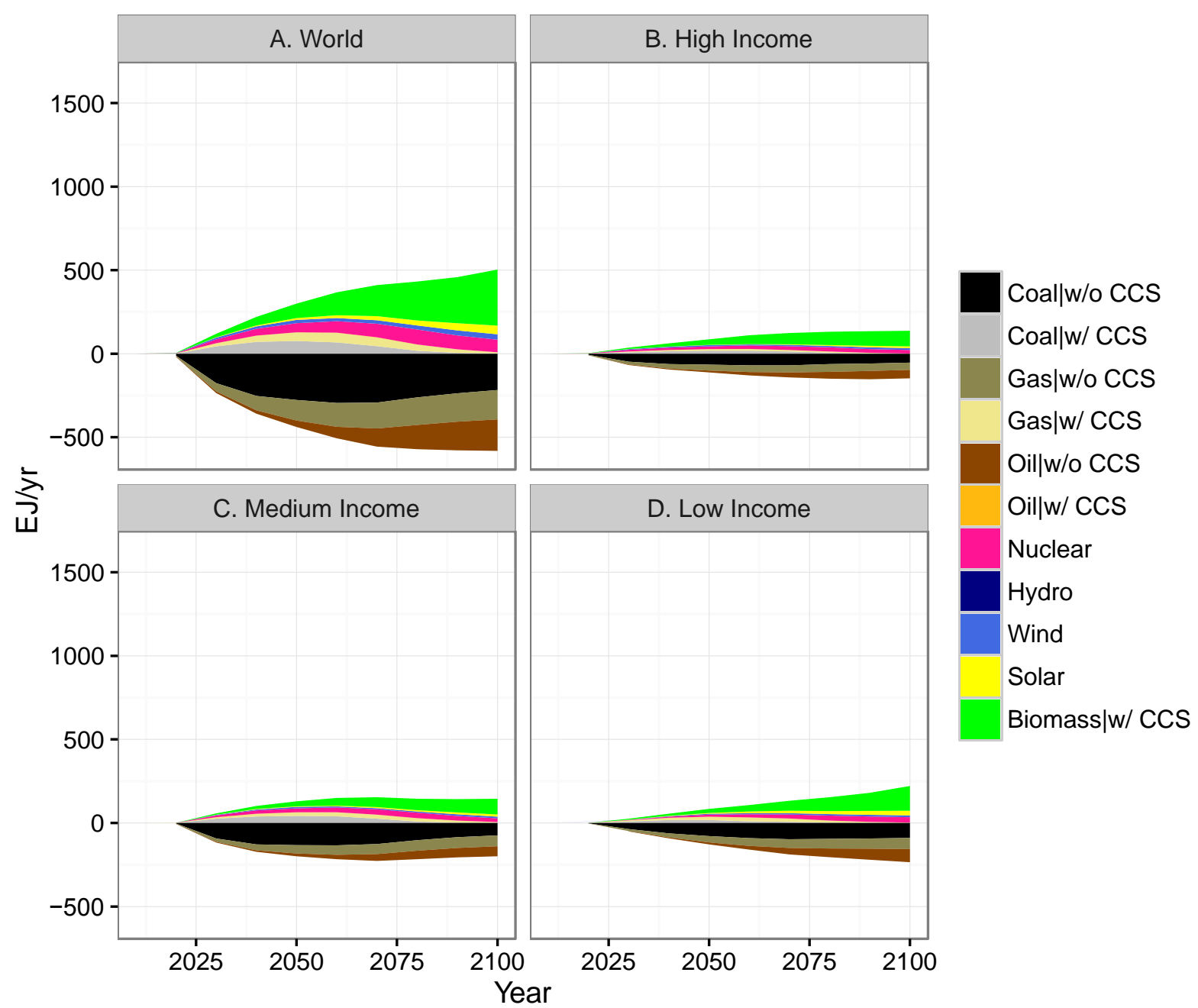

Figure 8: Change in Primary Energy Consumption by Income Group, between the SSP4 Baseline and SSP4 2.6 


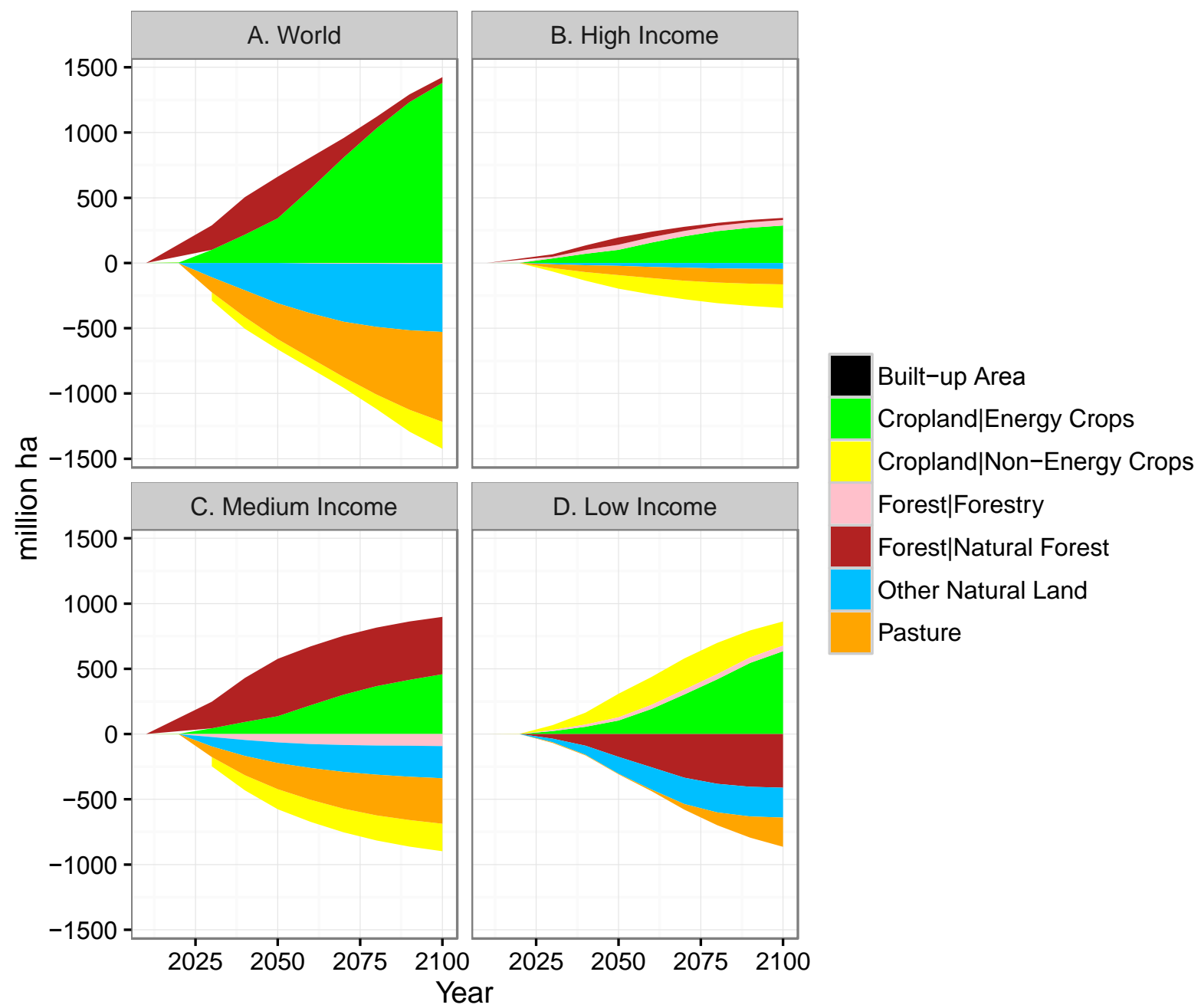

Figure 9: Change in Land Use by Income Group, between the SSP4 Baseline and SSP4 2.6 

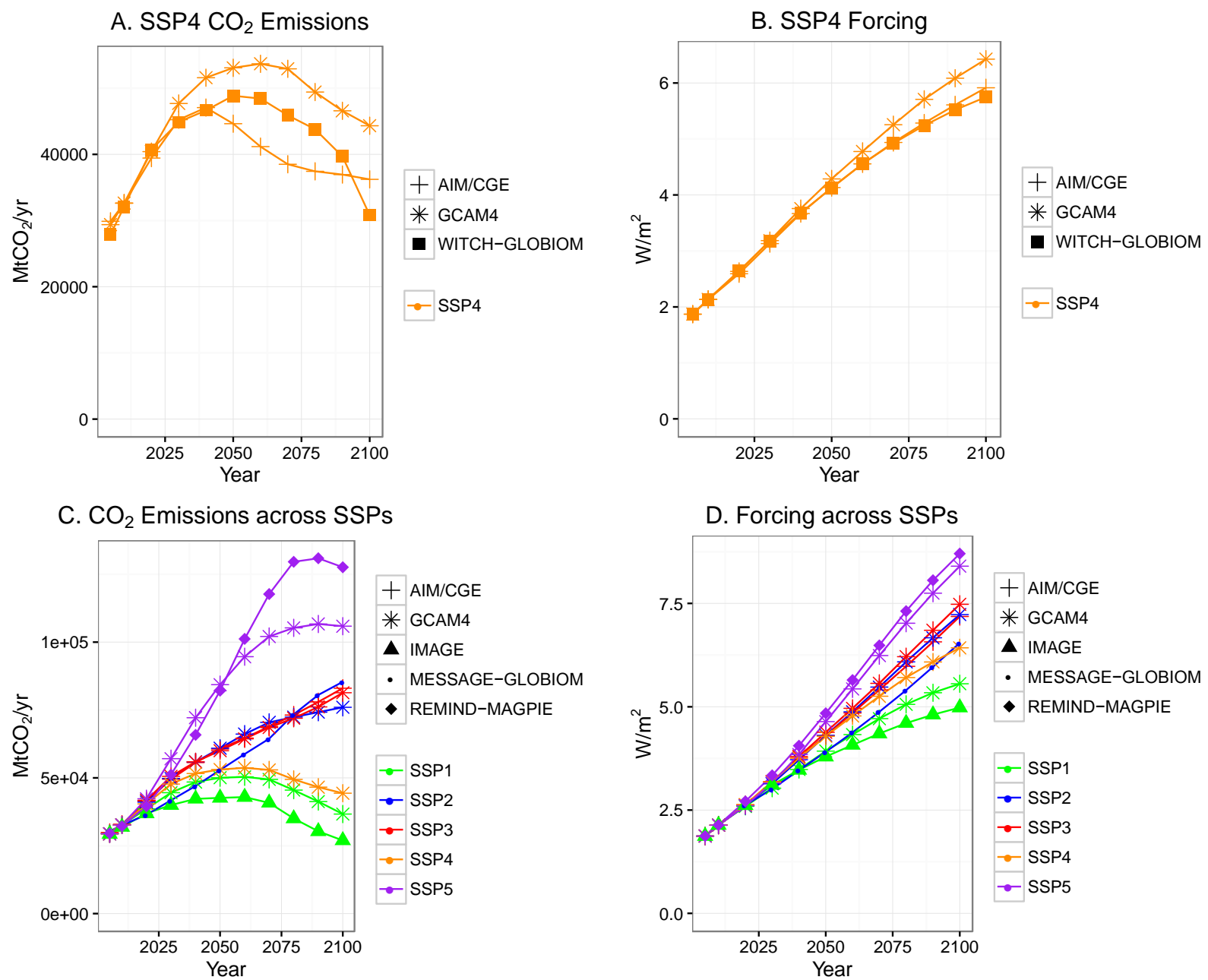

Figure 10: Comparison of the GCAM SSP4 Baseline to Other SSP4 Baseline Scenarios and other SSP Baselines 